\title{
ESTABLECIMIENTO DE SEIS ESPECIES ARBÓREAS NATIVAS EN UN PASTIZAL DEGRADADO EN LA SELVA LACANDONA, CHIAPAS, MÉXICO
}

\section{ESTABLISHMENT OF SIX NATIVE TREE SPECIES IN A DEGRADED PASTURE AT LACANDON RAINFOREST, CHIAPAS, MEXICO}

\author{
Francisco Román Dañobeytia ${ }^{1}$, Samuel Levy Tacher ${ }^{1}$, Hugo Perales Rivera ${ }^{1}$, Neptalí Ramírez Marcial ${ }^{1}$, David \\ Douterlungne $^{1}$ y Sergio López Mendoza ${ }^{1}$
}

Resumen

El presente estudio se realizó en un pastizal degradado en la Selva Lacandona, Chiapas, México. Se evaluó durante un año el efecto de tratamientos de deshierbe y fertilización, sobre el desempeño de plántulas de seis especies arbóreas nativas medido en términos de supervivencia y crecimiento. A partir de la época de sequía la probabilidad de supervivencia de las plántulas aumentó bajo la cobertura de los pastos (tratamiento sin deshierbe), tanto de especies demandantes de luz como tolerantes de sombra. Las plántulas de las especies de hábito heliófilo Ceiba pentandra, Schizolobium parahyba y Swietenia macrophylla tuvieron mayores niveles de supervivencia y crecimiento, con respecto a las umbrófilas Brosimum alicastrum, Calophyllum brasiliense y Ormosia schippii. La tasa relativa de crecimiento (TRC) en altura y diámetro varió significativamente para el efecto simple del deshierbe y de la especie. El efecto simple del deshierbe indicó un mayor crecimiento en altura y diámetro de las plantas en el tratamiento con deshierbe. No se registraron efectos significativos de la fertilización en el desempeño de las plantas. El efecto de la especie fue el factor más consistente para explicar la varianza en las tres variables de respuesta. Esto indica que las especies arbóreas estudiadas tuvieron determinadas trayectorias de supervivencia y crecimiento, al margen del efecto de los tratamientos aplicados.

Palabras clave: Desempeño de plántulas, deshierbe, fertilización, pastizales degradados, restauración ecológica, sucesión secundaria

\begin{abstract}
This research was carried out in a degraded pasture at Selva Lacandona tropical rain forest in Chiapas, Mexico. During a whole year weeding and fertilization effects were assessed on the seedling performance of six native tree species measuring survival and growth. From the dry season onwards the survival probability of the seedlings rise under grass covering (without weeding treatment) both for light demanding and shadow tolerating species. Helophytic seedlings, Ceiba pentandra, Schizolobium parahyba and Swietenia macrophylla had higher levels of survival and growth than shadow tolerating species, Brosimum alicastrum, Calophyllum brasiliense and Ormosia schippii. Relative growth rate (TRC) in height and diameter changed significantly for the simple effect of weeding and for the species itself. The simple effect of weeding indicated a higher growth on height and diameter of the plants under weeding treatment. We did not observe any significant effects of the fertilization on plants performance. The effect of the species was the most consistent factor in explaining variances in the three response variables, meaning that the tree species under investigation had specific survival and growth trajectories which lie aside the effect of the treatments.
\end{abstract}

Key words: Degraded pastures, fertilization, ecological restoration, seedling performance, secondary succession, weed control

\section{Introducción}

El trópico húmedo ha sufrido una deforestación extensa y acelerada a lo largo del siglo XX. A nivel mundial se había estimado que para el año 2 000, unas 850 millones de hectáreas (más de cuatro veces la superficie de México) se encontraban en estado de fragmentación y degradación (OIMT, 2 002). El proceso de deforestación suele iniciar con la extracción de madera comercial, a lo que le sigue el desmonte de la selva y la utilización del terreno por unos pocos años en agricultura de temporal, dedicándolo luego a pastizal permanente (Masera, 1996; Vásquez-Sánchez et al., 1992). La ausencia de cobertura arbórea, el pisoteo constante del ganado en suelos arcillosos y la elevada precipitación en las regiones tropicales húmedas, propician la degradación del suelo en términos de sus propiedades físicas y químicas. Entre ellas destacan la compactación del suelo, la lixiviación de nutrientes y la pérdida de la materia orgánica (Loker, 1994). La compactación del suelo por sobrepastoreo es una de las condiciones de degradación de mayor importancia en bosques y 
selvas de México, estimadas en un total 59 millones de hectáreas de tierras compactadas, que representa el $30 \%$ de la superficie total de tierras degradadas (Chapela, 1999).

Uno de los retos de la restauración ecológica en pastizales inducidos degradados, consiste en encontrar árboles nativos que puedan establecerse en estos suelos y que generen condiciones adecuadas para el desarrollo de la sucesión secundaria (Siemann \& Rogers, 2003; Lamb et al., 2005). En este sentido, diversos estudios señalan que la reintroducción de ciertas especies puede incrementar significativamente los niveles de materia orgánica y desencadenar el proceso de sucesión secundaria en áreas agropecuarias degradadas y abandonadas (Montagnini et al., 1995; Rhoades et al., 1998; Levy \& Golicher, 2004; Zahawi, 2005). Sin embargo, la limitada dispersión de semillas y las severas condiciones ambientales en los pastizales degradados pueden afectar el establecimiento de la vegetación arbórea (Holl et al., 2000; Zimmerman et al., 2000; Hooper et al., 2005). De igual forma, los efectos del manejo de las áreas agropecuarias tienen una influencia directa en las condiciones del suelo y la composición de la vegetación posterior al abandono de dichas áreas (Uhl, 1987; Levy, 2000; Ferguson et al., 2003), factores que condicionan el establecimiento de árboles nativos (Hooper et al., 2002; Zahawi, 2005).

La experimentación con especies nativas arbóreas o arbustivas empleando técnicas silvícolas ampliamente difundidas, como el deshierbe y la fertilización, constituyen prácticas sencillas e importantes para la restauración de vegetación arbórea en pastizales degradados tropicales (Holl et al., 2000; Long et al,. 2004). La meta del presente trabajo fue evaluar alternativas para el manejo de la sucesión secundaria, que faciliten y aceleren el establecimiento inicial de especies arbóreas nativas en un pastizal degradado. Con esta finalidad, se estableció un experimento para evaluar la respuesta de seis especies arbóreas nativas respecto a la aplicación de tratamientos de deshierbe y fertilización. Las especies utilizadas fueron Swietenia macrophylla King, Brosimum alicastrum Swartz., Ceiba pentandra (L.) Gaertn., Ormosia schippii Pierce ex Standl. \& Steyerm., Calophyllum brasiliense Cambess. y Schizolobium parahyba (Vell.) S.F. Blake.

\section{Materiales y métodos}

Área de estudio

La Selva Lacandona es el remanente más importante de selva húmeda tropical hacia el norte de Centroamérica, en la porción oriental del Estado de Chiapas (Nations et al., 1999). Esta región cubre una superficie aproximada de un millón de hectáreas en las cuencas de los ríos Jataté y Alto Usumacinta, siendo ésta última una de las fuentes de agua dulce más importantes de México.
El tipo de vegetación predominante es la selva alta perennifolia. Los árboles del estrato superior miden más de $30 \mathrm{~m}$ de alto y con cierta frecuencia alcanzan los 50 ó $60 \mathrm{~m}$, como en los casos de Guatteria anomala, Ceiba pentandra, Swietenia macrophylla, Terminalia amazonia y Ulmus mexicana (Pennington \& Sarukhán, 2005). Este ecosistema en gran parte de su extensión en esta región y en América Latina, se encuentra altamente fragmentado por áreas considerables de pastizales degradados, que retardan el desarrollo de la sucesión secundaria (Chapela, 1999).

El clima es cálido-húmedo con precipitaciones anuales promedio superiores a los $2000 \mathrm{~mm}$. La época principal de lluvia inicia en Junio y termina en Enero, y en Septiembre se da la mayor precipitación. Los meses más secos ocurren entre Enero y Mayo, siendo marzo el más seco con sólo $30 \mathrm{~mm}$ de promedio mensual. La temperatura promedio anual es de $24.7^{\circ}$ C (INE-SEMARNAP, 2000).

Suelo

El suelo donde se estableció la parcela experimental es arcilloso, deficiente en fósforo y con una alta densidad aparente $(1.1 \mathrm{~g} / \mathrm{ml})$, de acuerdo con el muestreo realizado durante el presente estudio. Este valor se incluye dentro del intervalo $(1.0-1.7 \mathrm{~g} / \mathrm{ml})$ definido como indicador de compactación en suelos arcillosos (Agüero \& Alvarado, 1983; Donahue et al., 1983). Durante los meses de mayor precipitación pluvial (Agosto-Septiembre) la parcela se encontró inundada, y por otro lado, en la estación seca se formaron grietas de aprox. $15 \mathrm{~cm}$ de profundidad. Batey \& McKenzie (2006) mencionan que el drenaje deficiente puede ser una consecuencia de la compactación en la superficie del suelo.

Riqueza florística inicial en el área experimental

De acuerdo con las colectas que se realizaron durante el estudio, se encontró que la especie dominante es un pasto introducido, conocido como 'estrella' Cynodon plectostachyus, principal fuente de alimento para el ganado vacuno en el potrero.

También se encontraron otras 37 especies de plantas, de las cuales más de la mitad (53\%) no son comestibles para el ganado, según el testimonio del propietario de la parcela. La familias más representadas son Asteraceae (23.7\%), Solanaceae y Poaceae (ambas 10.5\%) y Fabaceae (7.9\%). Las hierbas representan el 68\% del total de especies, los arbustos el 16\%, las trepadoras el 13\% y los árboles el $3 \%$.

Historial de uso del pastizal

El terreno fue ocupado como potrero durante 30 años a partir del desmonte de la selva. El sitio fue selva alta hasta el año de 1969, la cual fue tumbada y quemada para su aprovechamiento agrícola durante siete años. A partir del año de 1976 hasta la fecha, el área fue sembrada con pasto 'estrella' para su aprovechamiento pecuario. Al momento de realizar la 
presente investigación, el pastizal (de unas 25 ha, con cuatro divisiones) tenía un alto índice de agostadero (1.6 ha/unidad animal) lo cual es un indicador de baja productividad (SAGARPA, 2003). El manejo actual implica la limpieza o deshierbe con machete cada cuatro meses, con tres meses de descanso al año, tiempo durante el cual el propietario se ve obligado a alquilar otro potrero para que albergue su ganado, mientras el pasto de su terreno se recupera.

Selección de especies

El principal criterio para la selección de las especies fue que éstas pertenecieran a diferentes fases de la sucesión secundaria. Se incluyeron por un lado, especies heliófilas de semillas ortodoxas dispersadas por el viento, típicas de fases iniciales e intermedias de la sucesión. Por otro lado, se tomaron en cuenta especies umbrófilas de semillas recalcitrantes dispersadas por mamíferos, típicas de las fases tardías de la sucesión (Tabla 1).

Tabla 1. Características ecológicas y usos principales de las seis especies arbóreas seleccionadas.

\begin{tabular}{|c|c|c|c|c|}
\hline Especie & $\begin{array}{l}\text { Tipo de } \\
\text { semilla }^{1}\end{array}$ & $\begin{array}{c}\text { Agente } \\
\text { dispersor }^{2}\end{array}$ & $\begin{array}{l}\text { Necesidad } \\
\text { de luz }\end{array}$ & Usos $^{4}$ \\
\hline $\begin{array}{l}\text { Schizolobium } \\
\text { parahyba } \\
\text { FABACEAE }\end{array}$ & Ortodoxa & Viento & Heliófila & $\begin{array}{l}\text { Madera, } \\
\text { leña }\end{array}$ \\
\hline $\begin{array}{l}\text { Swietenia } \\
\text { macrophylla } \\
\text { MELIACEAE }\end{array}$ & Ortodoxa & Viento & Heliófila & Madera \\
\hline $\begin{array}{l}\text { Ceiba pentandra } \\
\text { BOMBACACEAE }\end{array}$ & Ortodoxa & Viento & Heliófila & $\begin{array}{l}\text { Fibra, } \\
\text { ritual }\end{array}$ \\
\hline $\begin{array}{l}\text { Brosimum } \\
\text { alicastrum } \\
\text { MORACEAE }\end{array}$ & Recalcitrante & $\begin{array}{c}\text { Murciélagos, } \\
\text { mamíferos }\end{array}$ & Umbrófila & $\begin{array}{c}\text { Madera, } \\
\text { leña } \\
\text { forraje }\end{array}$ \\
\hline $\begin{array}{l}\text { Ormosia schippii } \\
\text { FABACEAE }\end{array}$ & Recalcitrante & $\begin{array}{l}\text { Aves, } \\
\text { mamíferos }\end{array}$ & Umbrófila & $\begin{array}{l}\text { Madera, } \\
\text { artesanía }\end{array}$ \\
\hline $\begin{array}{l}\text { Calophyllum } \\
\text { brasiliense } \\
\text { CLUSIACEAE }\end{array}$ & Recalcitrante & $\begin{array}{c}\text { Murciélagos, } \\
\text { mamíferos }\end{array}$ & Umbrófila & Madera \\
\hline
\end{tabular}

1 Fuente: Smithsonian Tropical Research Institute (http://striweb.si.edu/esp/tesp/plant_species_c.htm)

${ }^{2,}$ Vazquez-Yanes et al., 1999

3,4 Pennington \& Sarukhán, 2005; Reynel at al., 2003

Establecimiento de la parcela experimental

El potrero seleccionado estaba en uso al iniciarse el presente estudio, por lo que la parcela fue cercada con alambre de púas para excluir al ganado del área experimental. Asimismo, se eliminó la vegetación superficial del potrero con el uso de machetes, para homogeneizar la cobertura inicial. Con la ayuda de cavadores se hicieron perforaciones en la tierra de aproximadamente $30 \times 30 \times 30 \mathrm{~cm}$ a intervalos de dos metros. El transplante a la parcela se realizó el día cinco de septiembre del año 2005, dentro del periodo principal de lluvias.El diseño experimental fue factorial en bloques completamente aleatorizados (DBCA), donde el deshierbe y la fertilización fueron los factores fijos, con dos niveles por cada factor (presencia y ausencia). Las parcelas y la posición de las especies en su interior fueron aleatorizadas en 15 bloques, cada uno con cuatro parcelas de 6 x $4 \mathrm{~m}$ y un individuo por especie a un distanciamiento de $2 \times 2$ m. Así, se instalaron en total 60 parcelas y fueron plantados 360 individuos en un área de $2226 \mathrm{~m}^{2}$.

Aplicación de tratamientos

El tratamiento de fertilización se inició al momento de la plantación, en la mitad de las parcelas designadas aleatoriamente en el diseño. En la base de hoyos de 20 × 20 × $30 \mathrm{~cm}$ se aplicó un fertilizante granular inorgánico comercial (N-P-K=20-30-10) en una dosis de $60 \mathrm{~g}$ por planta, el cual fue elegido por la deficiencia de fósforo en el suelo donde se estableció la parcela experimental.

El tratamiento de deshierbe se aplicó durante los primeros seis meses del establecimiento (Septiembre 2005 - Febrero 2006) a todos los individuos transplantados. Luego continuó los siguientes seis meses solamente en las parcelas con este tratamiento, realizándose una limpieza con machete alrededor de las plántulas en un radio de un metro. El deshierbe fue bimestral durante la temporada seca y mensual en tiempo de lluvias cuando crece a mayor velocidad.

\section{Evaluaciones}

La parcela fue evaluada cada 90 días. $\mathrm{La}$ primera evaluación se realizó al iniciar el experimento y se efectuaron un total de cinco mediciones a lo largo de un año. En cada una se evaluó la supervivencia y el crecimiento de las plantas. La supervivencia se registró mediante el conteo de los individuos y se anotaron las posibles causas de mortalidad (daños por insectos u hongos, desecación, daño mecánico). La altura de las plantas se obtuvo midiendo con un flexómetro desde la base del tallo hasta la yema apical. El diámetro basal se midió con un vernier calibrado con precisión de $0.05 \mathrm{~mm}$.

Análisis estadístico

El cambio en el crecimiento en altura y diámetro en el tiempo fue calculado a partir de la tasa relativa de crecimiento (TRC), la cual expresa el crecimiento en términos de la tasa de incremento en tamaño por unidad de tamaño inicial. La fórmula para calcular TRC es la siguiente:

$$
T R C=\log _{\mathrm{e}} H_{\mathrm{f}}-\log _{\mathrm{e}} H_{\mathrm{i}} / \mathrm{t}_{\mathrm{f}}-\mathrm{t}_{\mathrm{i}}
$$

donde $H_{\mathrm{f}}$ y $H_{\mathrm{i}}$ es la altura/diámetro en diferentes tiempos $\left(\mathrm{t}_{\mathrm{f}}-\mathrm{t}_{\mathrm{i}}\right)$ al final y al inicio del experimento.

La proporción de individuos sobrevivientes fue transformada para lograr una distribución normal de los datos. La fórmula es la siguiente:

$$
\mathrm{Y}_{2,1}=\operatorname{arcsen}\left(\mathrm{N}_{2} / \mathrm{N}_{1}\right)^{1 / 2}
$$


donde $\mathrm{N}_{2}$ y $\mathrm{N}_{1}$ es el número de individuos en el tiempo final con respecto a los que se establecieron en el inicio del experimento. primero resultó en que la supervivencia de las plantas fuera mayor en el tratamiento sin deshierbe (Figura 1), lo cual explica el por qué no se encontraron
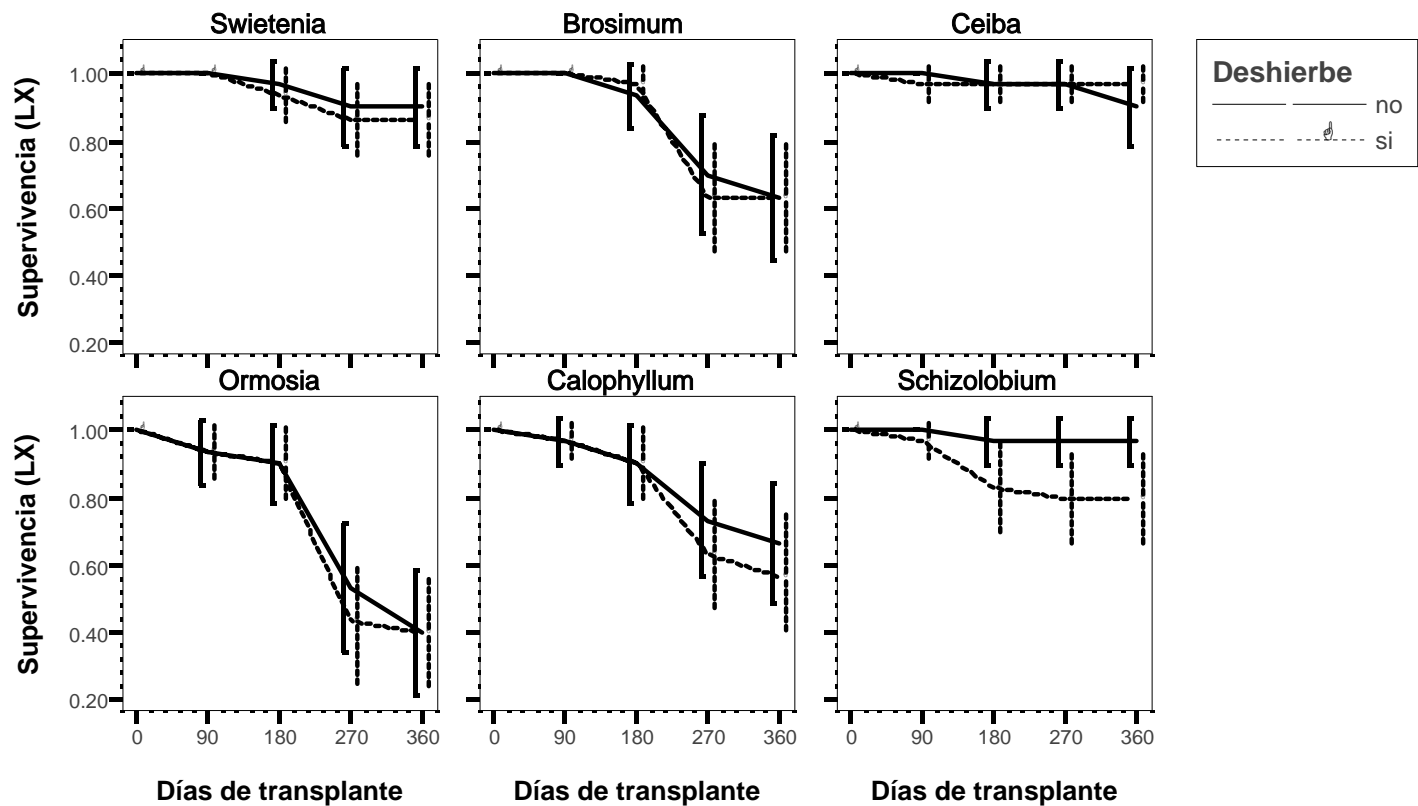

Figura 1. Efecto del tratamiento de deshierbe sobre la supervivencia (promedio \pm error estándar) de las seis especies a lo largo de un año.

Los datos fueron analizados mediante el análisis de varianza (ANOVA) univariante con dos niveles de análisis. En un primer nivel se evaluó la variación entre bloques, mientras que en el segundo nivel se analizó la variación por los efectos de tratamiento y especie. Cada nivel contó con una medida separada de la variación, por lo que los términos del error son presentados para ambas secciones del ANOVA. El análisis y las gráficas se realizaron con el programa estadístico SPSS (versión 11.0).

\section{Resultados}

Supervivencia

La supervivencia de las plantas al final del experimento varió significativamente entre bloques $\left(\mathrm{F}=2.4155, \mathrm{P}_{\mathrm{F}}=0.0032\right)$ y en la interacción de bloque con tratamiento $\left(\mathrm{F}=1.6839, \mathrm{P}_{\mathrm{F}}=0.0074\right)$. Esto implica que hubo mayor mortalidad en ciertos bloques, la cual se incrementó en la interacción con el deshierbe. Estas diferencias posiblemente se debieron a un efecto de gradiente ambiental relacionado con la compactación y humedad del suelo, generando en algunas zonas condiciones contrastantes de saturación durante la estación lluviosa y de sequedad extrema durante la estación seca.

También se encontraron diferencias significativas para el efecto simple del deshierbe $(F=5.339$, $\left.\mathrm{P}_{\mathrm{F}}=0.021\right)$ y de la especie $\left(\mathrm{F}=8.737, \mathrm{P}_{\mathrm{F}}<0.0001\right)$. Lo diferencias significativas en la interacción con la especie. Las especies más afectadas fueron $S$. parahyba $\left(\mathrm{F}=8.253, \quad \mathrm{P}_{\mathrm{F}}=0.004\right) \quad C$. brasiliense $\left(\mathrm{F}=3.176, \quad \mathrm{P}_{\mathrm{F}}=0.076\right)$ y $\quad$ O. schippii $(\mathrm{F}=3.052$, $\mathrm{P}_{\mathrm{F}}=0.082$ ). No se registraron efectos significativos por efecto de la fertilización.

En términos generales, la supervivencia fue buena en la época de lluvias y la mayor parte de la mortalidad (> 70\%) se dio entre los 180 y 270 días posteriores al transplante, periodo correspondiente con la estación seca y con la aplicación del tratamiento sin deshierbe (Figura 1). Un $20 \%$ de la mortalidad fue afectada por el corte accidental de la planta con machete al momento de aplicar el deshierbe y otro 10 $\%$ fue atribuido al estrés sufrido por las plántulas al momento del transplante. Los resultados indican que el establecimiento de las plántulas es bueno manteniendo el área deshierbada durante los primeros seis meses después del transplante (SeptiembreFebrero), y dejándolas sin deshierbe durante la época de sequía (Marzo-Junio).

\section{Crecimiento}

La tasa relativa de crecimiento (TRC) en altura varió significativamente para el efecto simple del deshierbe $\left(\mathrm{F}=6.6443, \mathrm{P}_{\mathrm{F}}=0.0106\right)$ y de la especie $\left(\mathrm{F}=221.076, \mathrm{P}_{\mathrm{F}}<0.0001\right)$. En cuanto a la TRC en diámetro basal se encontraron diferencias 
significativas por efecto del deshierbe $(\mathrm{F}=13.7807$, $\left.\mathrm{P}_{\mathrm{F}}=0.0003\right)$ y de la especie $\left(\mathrm{F}=318.1947, \mathrm{P}_{\mathrm{F}}<0.0001\right)$. No se registraron diferencias significativas para el efecto de bloque y tratamiento, ni para el de la fertilización. En ambos atributos de crecimiento la mayor parte de la varianza fue explicada por el efecto de la especie y en menor medida por el deshierbe.

El efecto simple del deshierbe indicó un mayor crecimiento en altura y diámetro de las plantas en el tratamiento con deshierbe (Figura 2). La diferenciación del crecimiento de las plantas por el deshierbe fue evidente a partir de los 180 días, fecha en que inició la aplicación del tratamiento sin deshierbe. En altura las respuestas más notorias fueron de $S$. parahyba $\left(\mathrm{F}=4.712, \mathrm{P}_{\mathrm{F}}=0.03\right)$ y $C$. brasiliense $\left(\mathrm{F}=3.136, \quad \mathrm{P}_{\mathrm{F}}=0.07\right)$. En diámetro las respuestas significativas también fueron de $S$. parahyba $\left(\mathrm{F}=5.279, \quad \mathrm{P}_{\mathrm{F}}=0.02\right)$ y $C$. brasiliense $(\mathrm{F}=6.651$, $\left.\mathrm{P}_{\mathrm{F}}=0.01\right)$, así como de $C$. pentandra $(\mathrm{F}=3.318$, $\left.\mathrm{P}_{\mathrm{F}}=0.06\right)$.

\section{Discusión}

Supervivencia

La principal causa de mortalidad de las plantas puede ser atribuida a la desecación, ya que durante la estación de menor precipitación (Marzo-Junio), se observó la formación de grietas en el suelo desnudo de las plantas con deshierbe. Estos resultados son consistentes con otros estudios que reportan que la mayor parte de la mortalidad de especies arbóreas que establecieron en zonas agropecuarias degradadas fue registrada durante la estación seca (Ramírez-Marcial, 2003; Hau \& Corlett, 2003; Alvarez-Aquino et al, 2004). Sin embargo, los resultados también pueden ser contrastantes con lo reportado usualmente sobre que el deshierbe favorece el establecimiento de plántulas forestales por la disminución de la competencia con los pastos (Holl et al., 2000; Zimmerman et al., 2000). El deshierbe si bien reduce la competencia también ocasiona que se hagan más extremas las condiciones de luminosidad, temperatura y falta de humedad, sobre todo durante la estación seca. Al quedar el suelo desnudo en la superficie, se incrementan los niveles de mortalidad por causa de la desecación (Nepstad et al., 1996; Hooper et al., 2002). Al respecto, Hernández-X. (1981) menciona que de acuerdo con el conocimiento agrícola tradicional maya del manejo del maíz, no es bueno el deshierbe en temporada de sequía porque las hierbas protegen al maíz del sol. En este sentido, nuestros resultados coinciden en que es posible que las especies umbrófilas tengan una mayor capacidad para sobrevivir bajo la cobertura de los pastos (Hooper et al., 2002; Rodríguez, 2005; Martínez-Garza et al., 2005). Sin embargo, de acuerdo con la respuesta de $S$. parahyba es posible que ante las severas condiciones de la estación seca, inclusive las especies demandantes de luz puedan ser favorecidas bajo una cobertura moderada de pastos.

\section{Crecimiento}

La mayoría de los estudios de establecimiento de árboles en pastizales señalan que la competencia de los pastos afecta negativamente el crecimiento de las plantas de especies arbóreas (Holl et al., 2000; Zimmerman et al., 2000; Hooper et al., 2005). La interacción biótica entre la cobertura de pastos y las plantas forestales puede darse a través de la interferencia lumínica y/o la competencia por recursos a nivel de las raíces (Davis et al., 1998; Huante et al., 1998). En nuestro estudio el efecto simple del deshierbe respalda esta consideración, ya que fueron beneficiadas las heliófilas $S$. parahyba y $C$. pentandra así como la umbrófila $C$. brasiliense.

Se observó un patrón de trueque o trade-off dado por el efecto del deshierbe, de forma negativa en la supervivencia y positiva en ambas variables de crecimiento. Este intercambio puede significar la pérdida de algunos individuos a cambio de alcanzar un mayor crecimiento a plena luz o viceversa. Según Baraloto et al. (2005) una forma de trueque, puede estar relacionada con el desempeño diferencial de una especie en microambientes contrastantes de luz o disponibilidad de agua. Estos canjes pueden tener una repercusión importante en la silvicultura y el manejo de especies forestales, así como en el entendimiento de la plasticidad y adaptación de las especies a ambientes perturbados (Poorter et al. 2005).

El no efecto de la fertilización puede responder a que su aplicación en condiciones saturadas pudo haber mermado su efecto por la disolución de sales, como también pudo haber contaminación entre parcelas y bloques. Al respecto Siemann \& Rogers (2003) mencionan que las propiedades físicas del suelo como la compactación pueden ser determinantes en la absorción de fertilizantes por plantas arbóreas. Es posible también que la composición del fertilizante rica en fósforo no haya sido la adecuada para dichas especies. Sin embargo, de acuerdo a las experiencias de Tanner et al. (1992) y Grogan et al. (2003), el efecto del fertilizante puede llegar a traducirse en crecimiento después de dos o tres años después de la aplicación.

Implicaciones para la restauración de pastizales degradados

Las condiciones de degradación en el área experimental, definidas en términos de compactación del suelo y deficiencia de algunos nutrimentos, historial de uso prolongado y baja productividad, no fueron un obstáculo infranqueable para el establecimiento de las plántulas. El manejo de la vegetación de pastizales mediante deshierbes selectivos, puede proveer varias alternativas en el establecimiento de especies nativas con diferente historia de vida y estatus sucesional (Davis et al., 1998). El entendimiento sobre el desempeño de una mayor diversidad de especies en diferentes escenarios perturbados es necesario para un escenario favorable a 

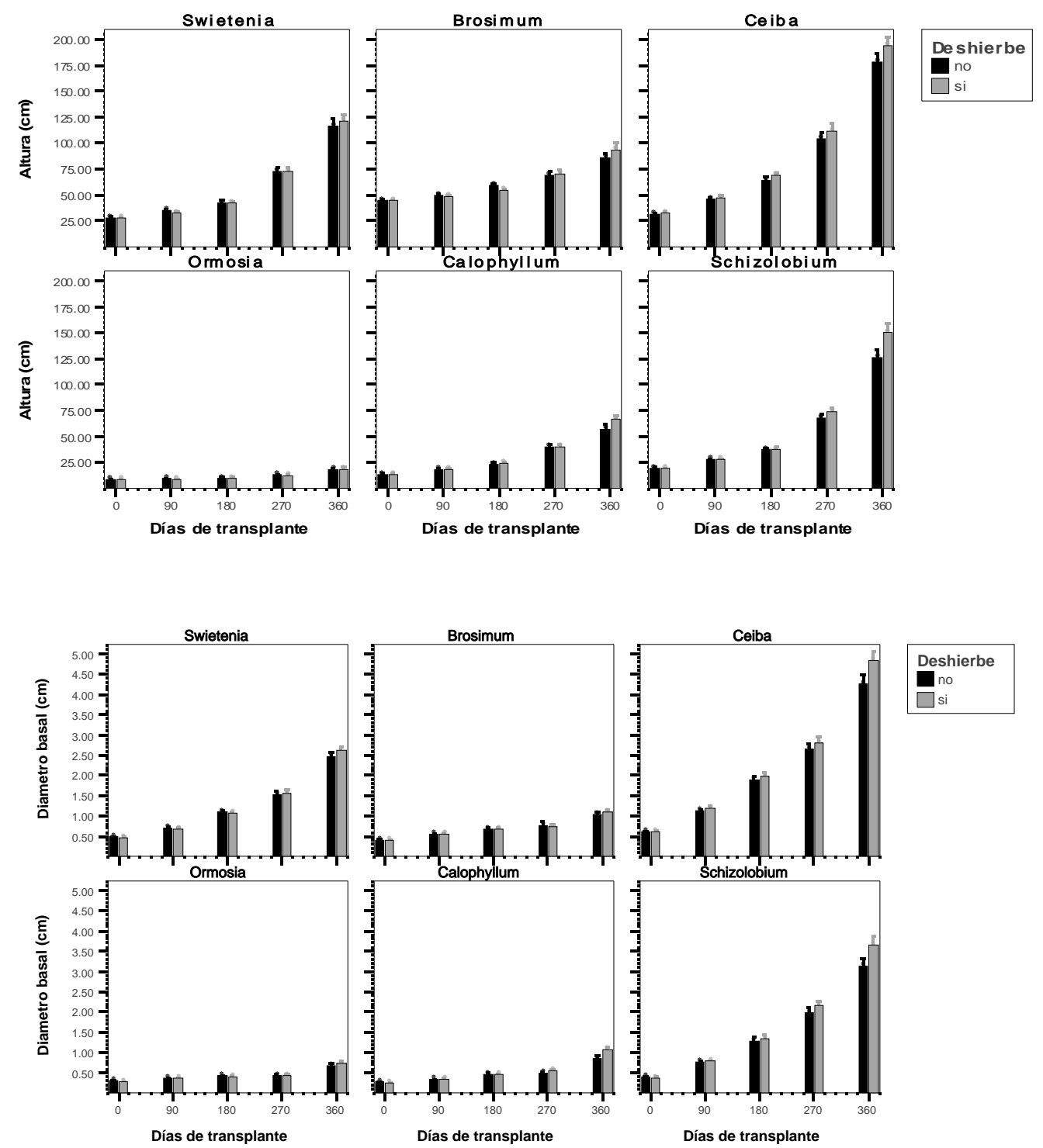

Días de transplante

Figura 2. Efecto del tratamiento de deshierbe sobre el crecimiento en altura y diámetro (prom. \pm e.e.) de las seis especies durante un año.

la restauración ecológica en áreas degradadas (Ramírez-Marcial, 2003). La selección de las especies más promisorias de acuerdo a las condiciones específicas de sitio, debe responder a criterios tanto ecológicos como socioeconómicos.

\section{Conclusiones}

La supervivencia de las plántulas es buena en épocas de alta precipitación (mes de Septiembre), sin la cobertura de pastos y manteniéndose con deshierbe a lo largo de la época de lluvias. A partir de la época de sequía (Marzo-Junio) la probabilidad de supervivencia de las plántulas es mayor bajo la cobertura de los pastos, tanto de especies demandantes de luz como tolerantes de sombra.

Las plántulas de las especies de hábito heliófilo Ceiba pentandra, Schizolobium parahyba y Swietenia macrophylla tuvieron altos niveles de supervivencia, mientras que las umbrófilas fueron más afectadas por la mortalidad, de manera intermedia en Brosimum alicastrum y Calophyllum brasiliense, y drásticamente en Ormosia schippii.

El efecto de la especie fue el factor más consistente para explicar la varianza en las tres variables de respuesta (supervivencia, crecimiento en diámetro y en altura). Esto indica que las especies 
arbóreas estudiadas tuvieron determinadas trayectorias de supervivencia y crecimiento en la condición ambiental en que fueron establecidas, al margen del efecto de los tratamientos aplicados.

\section{Agradecimientos}

A los pobladores de Lacanjá Chansayab y Nueva Palestina, por permitirnos trabajar en su comunidad, principalmente a Don Manuel Chank’in y Tomás Sánchez, dueños de las parcelas donde se produjeron las plántulas y se estableció la parcela experimental, respectivamente. A los lacandones Don Manuel Chankin, Héctor Chambor, Benjamín Chank’in y Adolfo Chank'in y al técnico Antonio Sánchez González por su invalorable apoyo durante el trabajo de campo. Esta investigación fue realizada gracias a becas otorgadas por la Comisión Nacional Forestal (CONAFOR) y el Consejo de Ciencia y Tecnología (COCYTECH).

\section{Literatura citada}

Agüero J. \& Alvarado A. 1983. Compactación y compactabilidad de suelos agrícolas y ganaderos de Guanacaste, Costa Rica. Agronomía Costarricense. 7(1/2): 27-33.

Alvarez-Aquino C., Williams-Linera G. \& Newton A. Experimental native tree seedling establishment for the restoration of a Mexican cloud forest. Restoration Ecology. 12(3): 412-418.

Baraloto C., Goldberg D. \& Bonal D. 2005. Preformance trade-offs among tropical tree seedlings in contrasting microhabitats. Ecology. 86(9): 2461-2472.

Batey T. \& Mc Kenzie D.C. 2006. Soil compaction: identification directly in the field. Soil Use and Management. 22: 123-131.

Chapela G. 1999. Causas estructurales de la degradación de tierras en México. En: Siebe C., Rodarte H., Toledo G., Etchevers J. \& Oleschko K. (Eds.). 1999. Conservación y Restauración de Suelos. Universidad Nacional Autónoma de México. México, D.F.

Davis M., Wrage A.K., Keith R. \& Reich R. 1998. Competition between tree seedling and herbaceous vegetation: support for a theory of resource supply and demand. Journal of Ecology. 86: 652-661.

Donahue R., Miller R. \& Shickluna J. 1983. Soils. An introduction to soils and plant growth. Fifth edition. Ed. Prentice-Hall, New Yersey.

Ferguson B., Vandermeer J., Morales H. \& Griffith D. 2003. Post-agricultural succession in El Peten, Guatemala. Conservation Biology 17(3): 818-828.

Grogan J., Ashton M. \& Galvao J. 2003. Big-leaf mahogany (Swietenia macrophylla) seedling survival and growth across a topographic gradient in southeast Pará, Brasil. Forest Ecology and Management. 186: 311-326.

Hau B.C. \& Corlett R.T. 2003. Factors affecting the early survival and growth of native tree seedlings planted on a degraded hillside grassland in Hong Kong, China. Restoration Ecology. 11(4): 483-488.

Hernández-X. E. 1981. Prácticas agrícolas. En: Várguez P., L.A. (Editor). La milpa entre los Mayas de Yucatán. : 45-73.
Holl K.D., Loik M.E., Lin E.H. \& Samuels I.A. 2000. Tropical montane forest restoration in Costa Rica: overcoming barriers to dispersal and establishment. Restoration Ecology. 8 (4): 339-349.

Hooper E., Condit R. \& Legendre P. 2002. Responses of 20 native tree species to reforestation strategies for abandoned farmland in Panama. Ecological Applications. 12(6): 1626-1641.

Hooper E., Legendre P. \& Condit R. 2005. Barriers to forest regeneration of deforested and abandoned land in Panama. Journal of Applied Ecology. 42: 1165-1174.

Huante P., Rincón E \& Chapin F.S. 1998. Foraging for nutrients, responses to changes in light, and competition in tropical deciduous tree seedlings. Oecologia. 117: 209-216.

INE-SEMARNAP. 2000. Programa de Manejo de la Reserva de la Biosfera Montes Azules, México. Instituto Nacional de Ecología - Secretaría de Medio Ambiente, Recursos Naturales y Pesca. México, D.F.

Lamb D., Erskine P. \& Parrota J. 2005. Restoration of degraded tropical forest landscapes. Science. 310: 16281632.

Levy S. \& Golicher J. D. 2004. How predictive is Traditional Ecological Knowledge? The case of the Lacandon Maya fallow enrichment system. Interciencia 29(9): 496-503.

Levy S. 2000. Sucesión causada por roza-tumba-quema en las selvas de Lacanhá Chansayab, Chiapas. Tesis de Doctorado. Colegio de postgraduados. Montecillos, Mexico.

Loker W.M. 1994. Where's the beef?: Incorporating cattle into sustainable agroforestry systems in the Amazon Basin. Agroforestry Systems. 25: 227-241.

Long J., Dean T. \& Roberts S. 2004. Linkages between silviculture and ecology: examination of several important conceptual models. Forest Ecology and Management. 200: 249-261.

Martinez-Garza C., Peña V., Ricker M., Campos A. \& Howe H. 2005. Restoring tropical biodiversity: Leaf traits predict growth and survival of late-successional trees in early successional environments. Forest Ecology and Management. 217: 365-379.

Masera O. 1996. Deforestación y degradación forestal en México. Grupo interdisciplinario de tecnología rural apropiada. Documento de trabajo $N^{\circ} 19$. Michoacán, México.

Montagnini F., Fanzeres A. \& Guimaraes da Vinha S. 1995. The potentials of 20 indigenous tree species for soil rehabilitation in the Atlantic forest region of Bahia. Journal of Applied Ecology. 32: 841-856.

Nations J.D., Primack R.B. \& Bray D. 1999. Introducción: La Selva Maya. En: Primack R.B., Bray D.B., Galletti H.A. \& Ponciano I. (Eds.). 1999. La Selva Maya. Conservación y Desarrollo. Ed. Siglo Veintiuno, México.: 13-20.

Nepstad D.C., Uhl C., Pereira C.A. \& Cardoso J.M. 1996. A comparative study of tree establishment in abandoned pasture and mature forest to eastern Amazonia. Oikos .76: 25-39.

OIMT. 2002. Directrices de la OIMT para la restauración, ordenación y rehabilitación de bosques tropicales secundarios y degradados. Serie de políticas forestales No. 13. Organización Internacional de las Maderas Tropicales. 
Pennington T.D. \& Sarukhán J. 2005. Árboles tropicales de México. Manual para la identificación de las principales especies. 3ra. Edición. Fondo de Cultura Económica. México, D.F.

Poorter L., Bongers F., Sterck F.J. \& Wöll H. 2005. Beyond the regeneration phase: differentiation of height-light trajectories among tropical tree species. Journal of Ecology. 93: 256-267.

Ramirez-Marcial N. 2003. Survival and growth of tree seedlings in antropogenically disturbed Mexican montane rainforests. Journal of Vegetation Science. 14: 881-890.

Reynel C., Pennington R.T., Pennington T.D., Flores C. \& Daza A. 2003. Árboles útiles de la Amazonía Peruana. Un manual con apuntes de identificación, ecología y propagación de las especies. Lima, 509 pp.

Rhoades C., Eckert G., \& Coleman D. 1998. Effect of pasture trees on soil nitrogen and organic matter: Implications for tropical montane forest restoration. Restoration Ecology. 6(3): 262-270.

Rodríguez J. 2005. Desempeño de plántulas transplantadas a praderas ganaderas abandonadas en la región de Marqués de Comillas, Chiapas. Tesis de Maestría en Ciencias. Universidad Nacional Autónoma de México. México, D.F.

SAGARPA. 2003. Secretaría de agricultura, ganadería, desarrollo rural, pesca y alimentación: http://www.sagarpa.gob.mx/dlg/chiapas/ganaderia/cotec oca

Siemann E. \& Rogers W. 2003. Changes in light and nitrogen availability under pionner trees may indirectly facilitate tree invasions of grasslands. Journal of Ecology. 91: 923-931.

Tanner E., Kapos V. \& Franco W. 1992. Nitrogen and phosphorus fertilization effects on Venezuelan montane forest trunk growth and litterfall. Ecology. 73(1): 78-86.

Uhl C. 1987. Factors controlling succession following slashand-burn agriculture in Amazonia. Journal of Ecology .75(2): 377-407.

Vásquez-Sánchez M.A., March I.J. \& Lazcano-Barrero M. A. 1992. Características socioeconómicas de la Selva Lacandona 1: 287-323. En: Vásquez-Sánchez M.A. \& Ramos M.A (Eds.). Reserva de la Biosfera Montes Azules, Selva Lacandona: Investigación para su conservación. Publ. Esp. Ecosfera, México.

Vázquez-Yanes C.; Batis Muñoz A. I.; Alcocer Silva M.I. Gual Díaz M. \& Sánchez Dirso C. 1999. Árboles y arbustos nativos potencialmente valiosos para la restauración ecológica y la reforestación. Reporte técnico del Proyecto J-084. Comisión Nacional de la Biodiversidad-Instituto de Ecología, Universidad Nacional Autónoma de México. Distrito Federal, México.

Zahawi R.A. 2005. Establishment and growth of living fence species: An overlooked tool for the restoration of degraded areas in the tropics. Restoration Ecology. 13(1): 92-102.

Zimmerman J.K., Pascarella J.B. \& Aide T.M. 2000. Barriers to forest regeneration in an abandoned Pasture in Puerto Rico. Restoration Ecology. (8)4: 350-360.

${ }^{1}$ El Colegio de la Frontera Sur. Carretera Panamericana y Periférico Sur s/n Barrio de María Auxiliadora CP 29290, San Cristóbal de las Casas, Chiapas. México, froman@posgrado.ecosur.mx, slevy@sclc.ecosur.mx, hperales@sclc.ecosur.mx, nramirezm@sclc.ecosur.mx, ddouterl@sclc.ecosur.mx, slmendoza@sclc.ecosur.mx 